\title{
L'échantillonnage dans l'étude de la minéralomasse forestière : l'exemple des taillis ardennais
}

\author{
C. MILLIER *, C. NYS ** et J. RANGER ** \\ * INRA, Mission informatique, Jouy-en-Josas \\ ** INRA, Station de Recherches sur les Sols forestiers \\ Champenoux, F 54280 Seichamps
}

\begin{abstract}
Résumé
La quantité de matière sèche et d'éléments minéraux exportée au cours de l'exploitation forestière est estimée pour 3 taillis d'espèces mélangées dans les Ardennes françaises. Des tarifs, régression linéaire, sont construits à partir d'échantillons.

Nous montrons dans cet article la nécessité d'établir des tarifs de biomasse ou de minéralomasse en fonction de chacun des facteurs étudiés. L'analyse de variance permet de classer, en fonction de la valeur du test $F$, les effets des facteurs ou de leur interaction soit : Espèce $\gg$ Compartiment $\gg>$ Station $>$ Espèce-Station $\triangleq$ Espèce-Compartiment $>$ Station-Compartiment.

La nécessité d'utiliser les tarifs spécifiques à chaque espèce, à chaque station et pour chacun des compartiments pose le problème de l'optimisation de l'échantillonnage. Nous admettons que la variance générale estimée à partir de nos échantillons est le meilleur estimateur de la variance générale. Nous calculons ainsi le nombre minimum d'individus (arbres) à la probabilité de 5 p. 100 pour satisfaire à une erreur de 10 p. 100 sur la moyenne.
\end{abstract}

Le nombre de 20 arbres échantillonnés par station et par espèce, se révèle insuffisant dans 17 p. 100 des cas.

Mots clés : Optimisation, échantillonnage, biomasse, minéraux, forêt, taillis.

\section{Introduction}

Le passage d'une sylviculture extensive à une sylviculture intensive pouvant aller jusqu'à l'exploitation totale de l'arbre pose le problème des conséquences de cette intensification sur la fertilité des stations.

Notre objectif est d'essayer d'évaluer les exportations d'éléments minéraux en fonction du degré d'intensification.

Les tarifs que nous mettons au point permettront d'utiliser les résultats de l'Inventaire Forestier National et ainsi d'ćvaluer pour une région donnée la récolte 
supplémentaire de biomasse en fonction du type d'exploitation et, en parallèle, le coût correspondant en éléments minéraux.

Afin de connaître les limites d'application de nos tarifs, il s'avère nécessaire d'étudier la variabilité due aux différents facteurs (espèce, station, arbre, etc.) entrant en jeu.

Un grand nombre d'études doit être envisagé. Il est donc souhaitable d'optimiser l'échantillonnage. Ce premier travail concerne l'étude de la variabilité dans trois taillis feuillus des Ardennes.

Ce genre d'étude a déjà été réalisć en particulier dans le domaine des analyses des sols. BEcketT \& Webster (1971) donnent une revue bibliographique de la variabilité des sols (méthodes analytiques, cartographie, etc.) et essayent de définir le nombre optimum des prélèvements. R. VAN DEN DRIESSCHE (1974) fait la revue des travaux concernant l'analyse des tissus végétaux dans ce même esprit.

Dans le domaine de la biomasse et de la minéralomasse des arbres forestiers, les travaux sont nombreux comme le montre la synthèse de F. CupI (1980). Certains auteurs ont étudié différentes espèces dans une même station, ou une même espèce sur différentes stations, mais il y a peu de conclusions spécifiques à l'échantillonnage et encore celles-ci sont contradictoires. Young \& CARPENTER (1976) proposent un échantillon moyen jugeant la variabilité minérale dans les parties de l'arbre comme faible. Par contre, Comerford \& LEAF (1982) préconisent un nombre d'arbres variable en fonction du seuil de signification souhaité.

C'est la raison pour laquelle nous pensons utile de résumer dans cet article nos premiers résultats.

\section{Matériel et méthodes}

\subsection{Les caractêristiques des taillis}

Les peuplements étudiés sont situés en forêt communale de Monthermé dans les Ardennes primaires. L'altitude moyenne est de $470 \mathrm{~m}$ et la pente quasi-nulle. Le climat de type montagnard est froid et humide. Les précipitations moyennes annuelles sont de $1250 \mathrm{~mm}$. La température moyenne annuelle est d'environ $8^{\circ} \mathrm{C}$.

Trois peuplements $(A, B, C)$ traités en taillis avec quelques très rares réserves ont été sélectionnés. L'inventaire a été réalisé dans chacun des peuplements à l'intérieur de deux types de placettes :

i) 10 placettes circulaires de 1 are distantes les unes des autres de $50 \mathrm{~m}$;

ii) une placette centrale de 10 ares qui a été divisée en 10 secteurs de 1 are; c'est dans ces 10 secteurs qu'ont été prélevés les arbres échantillons suivant une stratification globale pour l'ensemble de la parcelle centrale.

Le tableau 1 montre que trois espèces dominantes composent ce taillis : le Chêne (Quercus sessiliflora salisb.), le Bouleau (Betula verrucosa E.) et le Sorbier des oiseleurs (Sorbus aucuparia L.). Les arbres morts sont comptabilisés sans distinction d'espèces. 


\section{TABleau 1}

Inventaire (en pourcentage du nombre total d'arbres) par espèce pour le's 3 taillis.

Distribution of species in the 3 coppices ( $p .100$ of the total number of trees).

\begin{tabular}{|c|c|c|c|c|c|c|c|}
\hline & & $\begin{array}{l}\text { Nombre total } \\
\text { d'arbres/ha }\end{array}$ & Chêne & Bouleau & Sorbier & Divers & Morts \\
\hline Station & A $\ldots$ & 5210 & 26,7 & 16,4 & 14,5 & 1,9 & 40,5 \\
\hline Station & В $\ldots$ & 6380 & 28,9 & 18,2 & 7,5 & 4,0 & 41,4 \\
\hline Station & C $\ldots$ & 7635 & 31,7 & 21,3 & 13,5 & 2,7 & 30,8 \\
\hline
\end{tabular}

Les trois peuplements se différencient par le sol et l'âge. Le peuplement A est sur un sol brun acide sur limon épais $(60$ à $80 \mathrm{~cm})$ reposant sur les schistes primaires dont le pendage est proche de la verticale. Son âge est de 41 ans. En B, le sol est également un sol brun acide sur limon épais reposant sur les schistes, mais à littage horizontal. Le taillis B est âgé de 31 ans. Dans la station C le sol superficiel hétérogène varie du stagnogley au sol brun acide peu profond, développé dans 10 à $30 \mathrm{~cm}$ de limon sur les schistes. L'âge est aussi de 31 ans.

\subsection{Le plan d'échantillonnage}

Le plan d'échantillonnage a été déterminé pour répondre à l'objectif suivant :

Pouvoir évaluer les exportations minérales liées au type de sylviculture. La pratique usuelle d'exploitation des bois est celle de l'utilisation des bois de découpe supérieure à 7 ou $4 \mathrm{~cm}$ de diamètre. Actuellement, dans le but d'accroître la productivité des taillis, l'exploitation totale de l'arbre est envisagée. C'est la raison pour laquelle il est indispensable de connaître la biomasse et la composition chimique de la totalité du peuplement, y compris les petits bois.

Pour cela nous devons :

i) évaluer la minéralomasse des taillis et proposer des tarifs de calcul de cette minéralomasse en fonction du C 130 (circonférence à $1,30 \mathrm{~m}$ ) pour chacune des découpes usuelles;

ii) minimiser le coût des évaluations des minéralomasses en optimisant l'échantillonnage.

C'est pourquoi dans cette étude nous avons stratifié au maximum afin de pouvoir calculer les effets propres ou les interactions liées à : la station, l'espèce, le compartiment et l'arbre.

Dans les trois stations et pour les trois espèces dominantes ont été sélectionnés 20 arbres de manière à constituer un échantillon stratifié représentatif des diverses classes de surface terrière. Ce choix du nombre de 20 est un choix a priori, équilibre 
entre coût de l'étude et précision statistique. Il nous semblait suffisant pour établir les tarifs de biomasse et de minćralomasse avec une précision satisfaisante pour une utilisation pratique.

Chacun des arbres a été divisé en compartiment. Le compartiment est une unité homogène correspondant soit à un ensemble morphologique (exemple : les feuilles), soit à une unité d'utilisation commerciale du bois (exemple : la découpe $7 \mathrm{~cm}$ du tronc).

Les différents compartiments échantillonnés sont :

- les branches : feuilles et bois de branches;

- les troncs : bois et écorce.

Les gros bois, du tronc et des branches sont eux-mêmes séparés en trois découpes :

- la découpe supérieure à $7 \mathrm{~cm}$ de diamètre $\left(D_{7}\right)$;

- la découpe comprise entre 7 et $4 \mathrm{~cm}\left(\mathrm{D}_{1}\right)$;

- la découpe inférieure à $4 \mathrm{~cm}$ de diamètre $\left(\mathrm{D}_{0}\right)$.

\subsection{Les déterminations des éléments minéraux}

Les échantillons végétaux, après séchage à l'étuve à 65 "C, sont broyés et homogénéisés. L'azote, après minéralisation de type 'Kjeldahl' modifiée (acide sulfurique + catalyseur $\mathrm{K}_{2} \mathrm{SO}_{4}$, Se) est déterminé par colorimétrie au bleu d'indophénol (autoanalyseur Technicon). Les autres éléments sont déterminés après minéralisation en milieu perchlorique - eau oxygénée. Le calcium $(\mathrm{Ca})$ et le magnésium $(\mathrm{Mg}$ ) sont dosés par spectrométrie d'absorption atomique, le potassium par émission atomique, le phosphore est dosé par colorimétrie du complexe phosphovanadomolybdique.

\subsection{Evaluation de la biomasse et de la minéralomasse}

Les tarifs, suivant le modèle,

$\log [$ Bio $]=A+B \log [C$ 130]

$$
\text { C } 130=\text { circonférence }
$$

sont établis pour la biomasse et la minéralomasse. lls le sont au niveau du compartiment pour chacune des espèces et dans chacune des stations, à partir des données analytiques des branches ou des arbres échantillonnés.

Ces tarifs sont appliqués à l'inventaire des arbres (ou des branches).

L'on obtient ainsi pour chacun des 20 inventaires par site la quantité de matière sèche ou d'élément minéral répartic, pour chaque site, par espèce et par compartiment.

\subsection{Les techniques d'analyses statistiques}

Les analyses statistiques ont été effectuées sur les variables qualitatives (concentration en éléments dans les différents compartiments de l'arbre) puis sur les variables quantitatives correspondantes. 
Nous avons utilisé la programmathèque «AMANCE 81 » du Département de Biométric pour effectuer :

- les analyses statistiques courantes (moyennes, minima, maxima et ecarts-type) qui ont été exécutées systématiquement à tous les niveaux de l'échantillonnage:

- des analyses plus spécifiques qui sont :

\subsection{Les analyses de variance}

Deux modèles ont été utilisés. Le premier est une analyse de variance à 1 facteur de classification sur plusieurs variables. C'est le modèle le plus global qui peut s'écrire sous la forme :

$$
Y_{i j k}^{i v]}=u_{i j j}^{[v]}+\varepsilon_{i j k}^{[v]}
$$

Le deuxième modèle est l'analyse de variance non orthogonale sur des modèles à effets fixes. Seules les interactions d'ordre 1 sont calculées.

Pour les feuilles ou le bois des branches le modèle peut s'écrire :

$$
Y_{i j k}^{[v]}=i^{[v]}+\alpha_{j}^{[v]}+\beta_{j}^{[v]}+(\alpha \beta)_{i j}^{[v]}+\varepsilon_{j \mathbf{j} k}
$$

Pour le bois et l'écorce des troncs le modèle retenu est le suivant :

$$
Y_{i j l k}^{[v]}=l^{[v]}+\alpha \alpha_{i}^{[v]}+\beta_{j}^{[v]}+\gamma^{[v]}+(\alpha \beta)_{i j}^{[v]}+(\alpha \gamma)_{i j}^{[v]}+(\beta \gamma)_{j \mid}^{|v|}+\varepsilon_{i j l k}
$$

Les facteurs :

i : pour l'espèce

1: pour la découpe du tronc

$\mathrm{j}$ : pour la station

$\mathrm{k}$ : pour l'arbre

\subsection{Calcul du nombre d'échantillon}

L'ensemble des analyses statistiques précédentes laisse sans réponse l'un des points que nous jugeons primordial car il détermine le coût global de l'étude. Quel est l'effet «arbre » ct comment optimiser l'échantillonnage?

Une approche possible est l'évaluation à partir de l'intervalle de confiance, pour un scuil défini, du nombre minimal darbres (ou de branches, ou de sites).

soit $\mathrm{N}=\left(\frac{\mathrm{t} \cdot \sigma}{\mathrm{L}}\right)^{2} \quad$ où :

L : est ćgal à l'erreur consentie en pour cent de la moyenne,

$\sigma$ : est l'écart type calculé sur la base des échantillons prélevés,

$\mathrm{t}$ : a pour de valeur 1,96 au seuil de 5 p. 100. 


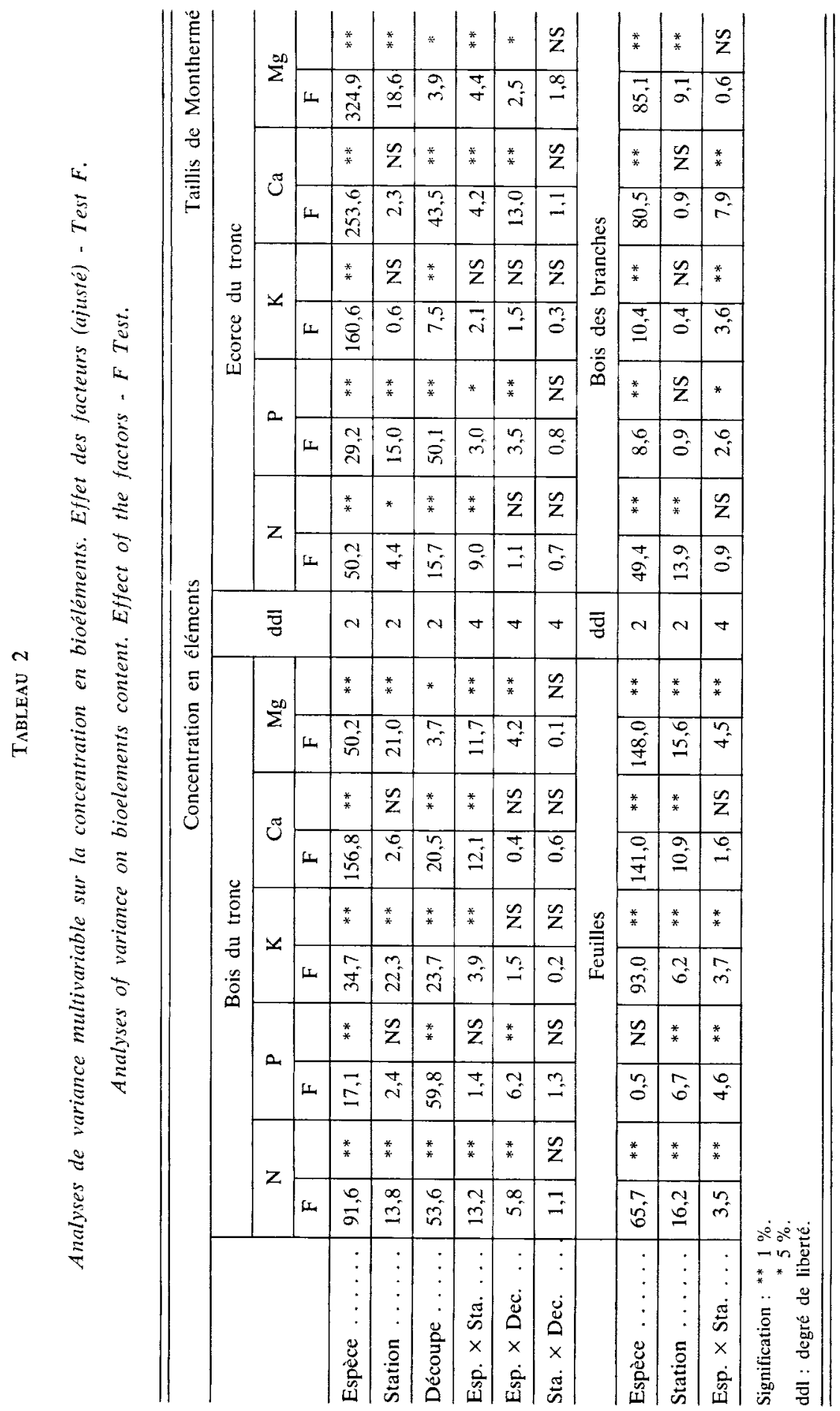




\section{Résultats}

Les résultats ici développés ont pour objet principalement l’analyse de variance. Nous résumons les résultats de la variabilité des concentrations ou des quantités des éléments majeurs : azote $(\mathrm{N})$, phosphore $(\mathrm{P})$, potassium $(\mathrm{K})$, calcium $(\mathrm{Ca})$, magnésium $(\mathrm{Mg})$, et, matière sèche.

\subsection{Etude qualitative}

Nous nous limitons dans cet article à l'évaluation des effets des facteurs : espèce, station et compartiment.

\subsection{Les branches}

Pour les deux compartiments bois des branches et feuilles, l'analyse de variance est limitée à deux facteurs et à leur interaction : station, espèce et interaction espècestation. Les résultats des calculs sont regroupés dans le tableau 2.

L'espèce est le facteur qui discrimine le plus à la fois les feuilles et le bois des branches. Le facteur station est discriminant pour les feuilles mais avec des valeurs du test $F$ très inférieures à celles du facteur espèce. Le facteur station n'est pas discriminant pour les éléments $\mathrm{P}, \mathrm{K}, \mathrm{Ca}$ du bois des branches. L'interaction espèce-station est faible, souvent proche du seuil de signification.

\subsection{Le tronc}

Pour les deux compartiments bois et écorce du tronc, l'analyse de variance a été réalisée pour les trois facteurs : espèce, station et découpe.

Les valeurs les plus élevées du test $F$ sont pour les facteurs espèce puis découpe. Pour le facteur station ce test n'est significatif que pour trois éléments $(\mathrm{N}, \mathrm{K}, \mathrm{Mg}$ pour le bois; M, P, Mg pour l'écorce).

En ce qui concerne l'interaction nous remarquons qu'entre station et découpe celle-ci est non significative ; l'interaction est faible entre espèce et découpe et significative entre espèce et station, sauf pour les éléments $\mathbf{P}$ du bois et $\mathbf{K}$ de l'écorce.

Pour l'ensemble des compartiments, il est possible d'établir une classification décroissante à partir des valeurs du test $F$, de l'effet des facteurs ou des interactions. Espèce $>>$ Découpe $>>>$ Station $>$ Espèce-Station $\geqslant$ Espèce-Découpe $>$ StationDécoupe.

\subsection{Etude quantitative}

Les quantités d'éléments par placette, pour chacun des compartiments et par espèce résultent de l'application de tarifs. Ceux-ci ont pour modèle une équation de type :

$$
\log [\text { M.S. } \times \text { El. }]=\mathrm{Ai}+\mathrm{Bi} \log [\mathrm{C} 130] *
$$

* M.S. = Matière sèche à $65^{\circ} \mathrm{C}$.

El. = Concentration en élément chimique en p. 100

C130 $=$ Circonférence de l'arbre à $1,30 \mathrm{~m}$ de hauteur en $\mathrm{cm}$. 
Tableau 3

Analyse de variance multivariable à plusieurs facteurs.

Quantité de M.S. et de bioéléments - test F.

Multivariate analysis with several factors. Dry weight and mineral elements - F test.

\begin{tabular}{|c|c|c|c|c|c|c|c|c|c|c|c|c|}
\hline & \multicolumn{2}{|c|}{ M.S. } & \multicolumn{2}{|c|}{$\mathrm{N}$} & \multicolumn{2}{|c|}{$P$} & \multicolumn{2}{|c|}{ K } & \multicolumn{2}{|c|}{$\mathrm{Ca}$} & \multicolumn{2}{|c|}{$\mathrm{Mg}$} \\
\hline & \multicolumn{12}{|c|}{ Feuilles } \\
\hline Espèce . & 102,9 & $* *$ & 145,4 & $* * *$ & 138,5 & $* *$ & 134,7 & $* *$ & 125,7 & $* *$ & 136,1 & $* *$ \\
\hline Station ..... & 3,6 & * & 1,9 & NS & 4,7 & $* *$ & 4,3 & * & 27,0 & $* *$ & 5,5 & $* *$ \\
\hline \multirow[t]{2}{*}{ Esp. $\times$ Stat. } & 1,3 & NS & 2,5 & * & 5,25 & $* *$ & 5,2 & $* *$ & 25,2 & $* *$ & 3,2 & $* *$ \\
\hline & \multicolumn{12}{|c|}{ Bois des branches } \\
\hline Espèce & 75,4 & $* *$ & 141,7 & ** & 128,6 & $* *$ & 139,5 & $* *$ & 113,0 & $* *$ & 112,9 & $* *$ \\
\hline Station ..... & 0,8 & NS & 2,4 & NS & 3,4 & $*$ & 6,2 & $* *$ & 0,8 & NS & 4,6 & $*$ \\
\hline \multirow[t]{2}{*}{ Esp. $\times$ Stat. $\ldots$} & 0,3 & NS & 1,3 & NS & 4.1 & $* *$ & 5,0 & $* *$ & 1,3 & NS & 1,4 & NS \\
\hline & \multicolumn{12}{|c|}{ Tronc $(b+e) D_{7}$} \\
\hline Espèce & 125,5 & $* *$ & 131,4 & $* *$ & 118,0 & $* *$ & 134,4 & $* *$ & 157,7 & $* *$ & 111,7 & $* *$ \\
\hline Station ...... & 13,7 & $* *$ & 15,1 & $* *$ & 6,7 & $* *$ & 4,6 & $*$ & 7,0 & $* *$ & 20,9 & $* *$ \\
\hline \multirow[t]{2}{*}{ Esp. $\times$ Stat. $\ldots$} & 6,4 & $* *$ & 7,3 & $* *$ & 3,9 & $* *$ & 2,4 & * & 4,0 & $* *$ & 7,3 & ** \\
\hline & \multicolumn{12}{|c|}{ Tronc $(b+c) D_{4}$} \\
\hline Espèce & 151,3 & $* *$ & 88,2 & ** & $\mathbf{P} 49,9$ & $* *$ & 171,5 & ** & 185,4 & $* *$ & 137,4 & $* *$ \\
\hline Station & 11,3 & $* *$ & 9,2 & ** & 3,8 & * & 4,1 & $*$ & 3,7 & * & 14,0 & $* *$ \\
\hline \multirow[t]{2}{*}{ Esp. $\times$ Stat. } & 5,0 & $* *$ & 5,7 & $* *$ & 2,4 & * & 1,5 & NS & 2,5 & $*$ & 4,8 & $* *$ \\
\hline & \multicolumn{12}{|c|}{ Tronc $(b+e)$ Total } \\
\hline Espèce & 158,7 & $* *$ & 174,3 & ** & 157,9 & $* *$ & 186,5 & $* *$ & 192,7 & $* *$ & 128,5 & $* *$ \\
\hline Station & 9,5 & $* *$ & 9,5 & $* *$ & 2,0 & NS & 8,7 & $* *$ & 2,5 & NS & 12,6 & $* *$ \\
\hline \multirow[t]{2}{*}{ Esp. $\times$ Stat. } & 4,6 & $* *$ & 5,4 & $* *$ & 1,8 & NS & 3,9 & $* *$ & 2,3 & $*$ & 3,1 & $* *$ \\
\hline & \multicolumn{12}{|c|}{ Arbre total ligneux } \\
\hline Espèce ..... & 156,9 & $* *$ & 167,9 & $* *$ & 148,8 & $* *$ & 170,1 & $* *$ & 172,5 & $* *$ & 133,7 & $* *$ \\
\hline Station & 5,0 & $* *$ & 4,7 & $*$ & 0,6 & NS & 0,2 & NS & 0,9 & NS & 7,8 & $* *$ \\
\hline Esp. $\times$ Stat. $\ldots$ & 2,5 & $*$ & 1,8 & NS & 0,8 & NS & 0,1 & NS & 1,5 & NS & 2,6 & * \\
\hline
\end{tabular}


Ce modèle nous a donné les résidus les plus faibles. Son biais peut être corrigé (BASKERVILLE, 1972).

L'inventaire avait été réalisé sur deux types de placettes. Soit des placettes circulaires d'un are, soit des placettes triangulaires correspondant au dixième de la placette centrale de 10 ares. La comparaison entre ces deux types de placettes ne montre pas de différence significative (Bouchon et al., 1985).

C'est la raison pour laquelle dans la suite de l'étude nous effectuerons les analyses de variance sur les résultats confondus des deux types de placettes.

Les résultats de l'analyse de variance à deux facteurs : espèce et station sont les suivants :

- quel que soit le compartiment, l'espèce est le facteur qui différencie le mieux les quantités des différents éléments minéraux (tabl. 3). L'effet station est très peu marqué et variable d'un compartiment à l'autre et d'un élément à l'autre sauf en ce qui concerne les découpes à $7 \mathrm{~cm}$ (D4), nous constatons qu'il y a un effet significatif du facteur station;

- l'interaction Espèce $\times$ Station existe dans 50 p. 100 des cas mais sans loi générale.

En résumé, pour l'aspect quantitatif comme pour l'aspect qualitatif le facteur espèce est le factcur le plus discriminant.

\subsection{Optimisation de l'échantillonnage}

Les résultats de la première partie de cet article démontrent qu'il existe des différences tant au point de vue qualitatif que quantitatif. Il faut donc tenir compte de ces différences et analyser séparément, dans chacune des stations choisies, chaque espèce et chacun des compartiments représentant une unité morphologique de l'arbre.

Le plan d'échantillonnage doit être établi en fonction de ces résultats. Cependant le coût financier de telles études est élevé et lié principalement au coût des analyses qualitatives.

Est-il possible d'optimiser ce plan d'échantillonnage? C'est-à-dire réduire le nombre des arbres (ou des branches) échantillons en conservant une valeur de l'erreur compatible avec les attendus de l'étude.

Trois des facteurs sont imcompressibles : Station, Espèce et compartiment.

Nous ne devons donc prendre en considération que les individus des populations : le nombre des arbres (ou des branches) analysés pour le qualitatif, le nombre des parcelles inventorices dans le cas du quantitatif.

L'étude des liaisons entre le C130 et la composition élémentaire d'un tissu montre qu'il n’y a pas de loi générale : il existe, ou il n'existe pas, une liaison linéaire significative, positive ou négative, avec le paramètre mesuré (C130).

S'il y a relation linéaire, celle-ci peut s'écrire suivant le modèle $\mathrm{Y}=$ ao + al $(\mathrm{C} 130)+\varepsilon$. 


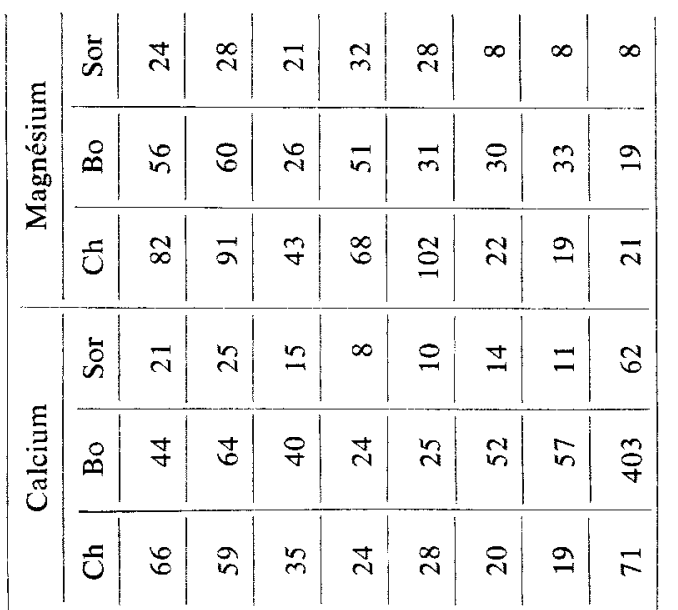$$
\dot{2}
$$

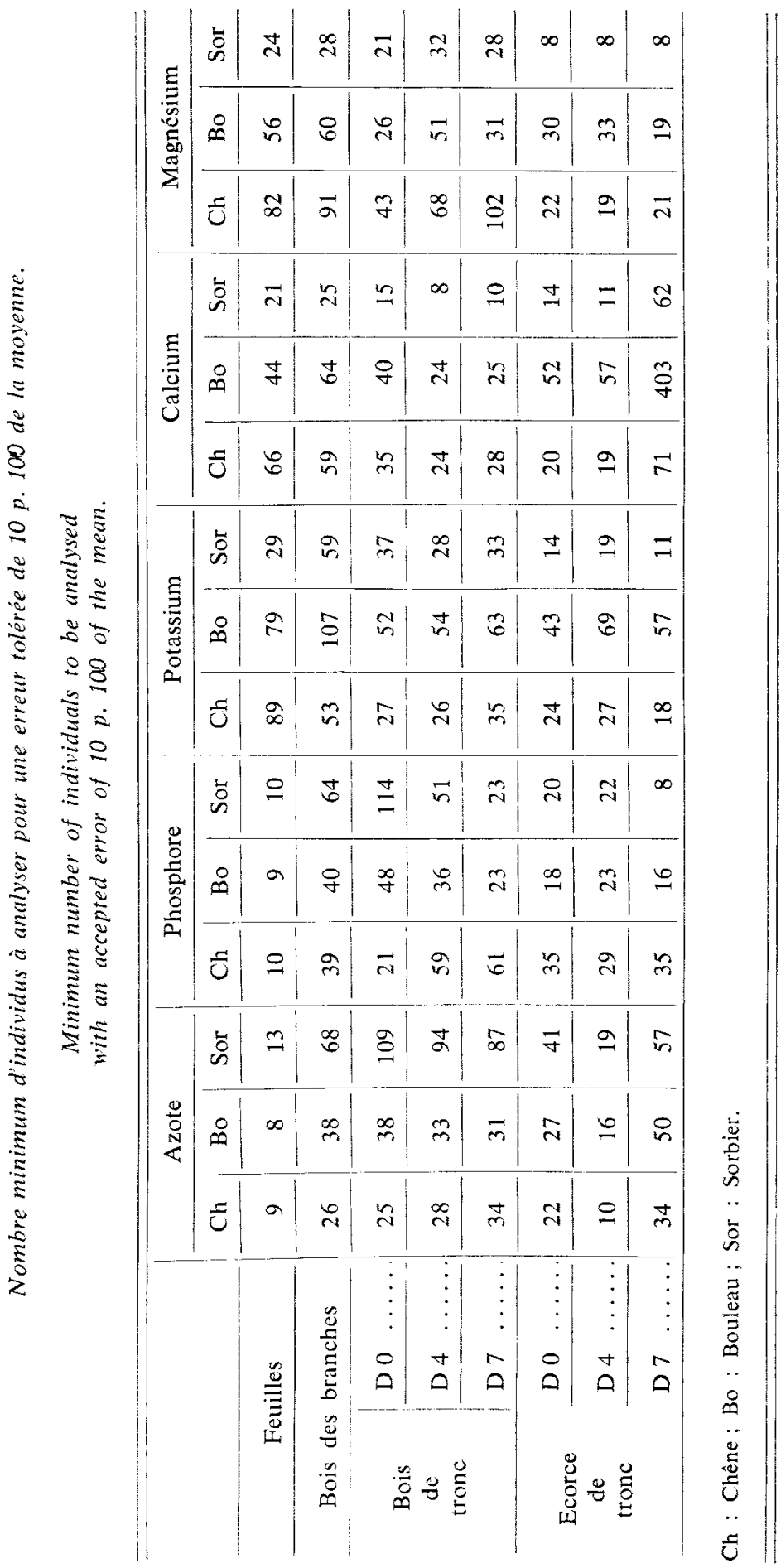


Pour optimiser l'échantillonnage il faut rendre minimale la valeur Var al (si $\mathrm{C} 130=\mathrm{x})$ soit $\operatorname{Var} \mathrm{a} 1=\frac{\sigma^{2}}{\Sigma(\mathrm{xi}-\overline{\mathrm{x}})^{2}}$ minima. Cela implique que $\Sigma(\mathrm{xi}-\overrightarrow{\mathrm{x}})^{2}=$ $\sum \mathrm{xi}^{2}-\mathrm{n} \overline{\mathrm{x}}^{2}$ soit maximum. Cest le cas $\mathrm{i} \mathrm{n} / 2$ points sont choisis aux minima et maxima.

Mais nous ne savons pas a priori, si la liaison existe. De ce fait seul le cas général où il n'existe pas de liaison peut être étudié.

Dans la partie analytique de ce travail nous avons fait des estimations de la variance générale et nous pouvons utiliser ce résultat en posant pour hypothèse que cette estimation est la meilleure valeur de celle-ci.

Le nombre minimum d'individus peut êtire calculé suivant la formule

$$
\mathbf{N}=\left[\frac{1,96 \times \sigma}{\mathrm{L}}\right] \text {. }
$$

La valeur de $\sigma$ est l'estimation de la variance générale. Et dans cette étude il est raisonnable de se fixer un intervalle de confiance $L$ égal à 10 p. 100 de la moyenne. l'arbre.

Le tableau 4 donne les résultats de ce calcul pour les différents compartiments de

On constate que le nombre d'échantillons à analyser est très variable d'un compartiment à l'autre et d'un élément à l'autre. De plus la variabilité est plus grande pour l'espèce Sorbier alors que Bouleau et Chêne sont voisins.

Dans notre cas le choix a priori de 60 arbres par espèce se révèle insuffisant dans 17 p. 100 des cas.

\section{Conclusion}

Nous avions fait un choix a priori en sélectionnant 3 stations dans les Ardennes se différenciant par le type de sol et l'âge. Les analyses statistiques réalisćes à partir de notre échantillonnage ont montré que tant du point de vue qualitatif que quantitatif, ces trois stations, en général, se différencient les unes des autres; la station $\mathbf{C}$. s'écartant le plus de la moyenne.

Notre méthode d'analyse et le nombre d'individus échantillonnés permettent déjà de distinguer les stations entre elles.

Nous devons aussi savoir jusqu'où il est nécessaire de pousser l'analyse.

Le taillis comporte 3 espèces. Ce facteur s'est révélé le plus discriminant des facteurs étudiés et beaucoup plus que le facteur station.

Il est donc impératif dans toute étude de minéralomasse d'échantillonner séparément les espèces. 
Selon les objectifs de l'étude il faut ou non compartimenter l’arbre. Il était $a$ priori évident de distinguer les divers compartiments morphologiques (à comportement physiologique différent). Mais était-il judicieux de séparer les différentes découpes commerciales du tronc?

Les analyses statistiques ont confirmé les différences entre compartiments morphologiques. Elles ont démontré de plus que les découpes commerciales sont différentes. Les découpes $D_{4}$ et $D_{\tau}$ sont de composition minérale voisine, mais elles sont différentes de la découpe «fin bout » $D_{n}$ qui est celle du bois jeune.

Chaque unité doit donc être échantillonnée séparément.

Le coût final d’une telle étude dépendra ainsi pour une unité définie, du nombre d'échantillons prélevés; c’est-à-dire du nombre des branches, des arbres et des placettes.

Que faut-il conclure de l'optimisation de l'échantillonnage?

Le chapitre «effet arbre propose le nombre d'individus nécessaire aux conditions de seuil que nous nous sommes fixćes.

En pratique, le nombre d’individus idéal dépasse les moyens crédibles pour de tels travaux. Il nous faut done envisager les différentes solutions qui suivent :

1. Prendre comme le proposent Young \& Carpenter (1976) l'échantillon moyen. Mais comment le définir lorsque nous venons de montrer que l'échantillon moyen change avec le compartiment et l'élément et que dans les taillis la variabilité est importante (Bouchon et al., 1985).

2. Faire un échantillon moyen-pondéré en prélevant un pourcentage représentatif, par exemple le proportionnel à la matière sèche.

3. Suivre Ie plan d’échantillonnage proposé dans ce travail mais être conscient que prélever 30 branches donnera une estimation à 10 p. 100 de la moyenne et que prélever et analyser 15 arbres, par espèce et par station permettra une estimation à 15 p. 100 de la moyenne.

La première solution n’est plus recommandable compte tenu de ce qui a été acquis comme connaissance sur la variabilité de composition des tissus végétaux.

La deuxième solution ne permet qu'une évaluation quantitative et elle peut être suffisante pour estimer les exportations d'une exploitation.

La troisième solution, plus coûteuse, permet en plus de parfaire nos connaissances sur la variabilité des compositions et d'optimiser ainsi le type d'exploitation.

Reca en mars 1985.

Accepté en juillet 1985. 


\section{Summary}

\section{Sampling in a forest mineralomass study: using an example from three coppices in the Ardennes}

The intensification of forestry (including whole tree harvesting) raises the question of its consequences on site fertility. We evaluate the removal of mineral elements related to the degree of intensification by applying tariffs established by stratified sampling.

Here we summarise the limitations to the application of these tariffs due to the variability of other factors : species, site, tree and its components.

- Three coppices in the Primary Ardennes were sampled by :

1. Species : Oak (Quercus sessiliflora), birch (Betula verrucosa), and mountain ash (Sorbus aucuparia). shales.

2. Site : "sol brun acide» with greater $(80 \mathrm{~cm})$ or lesser $(40 \mathrm{~cm})$ depths of loess over

3. Tree component : leaves, branches, and three parts of the trunk cut at $7 \mathrm{~cm}, 4 \mathrm{~cm}$ and $\mathrm{l} \mathrm{cm}$ diameters.

- The dry weight of the material at $65^{\circ} \mathrm{C}$ and major elements, N, P, K, Ca, My were determined.

We used the following tariff model :

$\log$ (biomass) $=a+b \log ($ circumference)

and applied the correction for bias as used by Baskerville (1972).

- The analyses of variance with one or more factors were measured to examine the effects of the factors. We used analysis of co-variance to compare the tariffs.

Results :

The qualitative examination of the variation in the chemical composition of the different components, showed that each is partly a function of site, but is primarly due to species (table 2). The interaction between species and site is low. We can classify the effects of, and interactions between, factors as a function of their $F$ test value in the following order of decreasing magnitude: Species $>>$ tree component $\gg>$ site $>$ species $x$ site species $\times$ component $>$ station $\times$ component.

The quantitative results (table 3 ) confirm that species is the most discriminating factor.

There are also significant differences between the tariffs. These must be specific to species, site and tree component.

The cost of these studies is high, so it was not possible to regroup the samples. Therefore, is it possible to increase the efficiency of the sampling strategy?

As no general law exists for the relationship between the parameter measured (circumference at $1.30 \mathrm{~m}$ or $\mathrm{C} 130$ ) and the mineral content of tree tissue, we calculated the minimum number of individuals, $N$, necessary by using the formula :

$$
\mathrm{N}=\left[\frac{1.96+\sigma}{\mathrm{L}}\right]^{2}
$$

at $5 \mathrm{p}$. 100 where $\mathrm{L}$ is the confidence limit at $10 \mathrm{p}$. 100 of the mean. The value of $\sigma$ is the value of the variance, and we admit that the best estimate is the variance estimated from our samples.

Table 4 shows the results calculated for the different tree components. The variability is greater for mountain ash than for birch or oak.

In our study, the choice of 60 trees per species is theoretically insufficient in $17 \mathrm{p} .100$ of the cases. At each site, for each species, we propose to sample 30 branches to estimate the mean at 10 p. 100 , and 15 trees for the mean at 15 p. 100 .

Key words : Optimization, sampling, biomass, mineral content, forest, coppice. 


\section{Références bibliographiques}

Bachacou J., Masson J.P., Millier C., 1981. Manuel de la programmathèque statistique. Amance. CNRF-INRA, 54280 Champenoux, France.

Baskerville G.L., 1972. Use of logarithmic regression in the estimation of plant biomass. Can. J. For., 2, 49.

Becketr P., Webster R., 1971. Soil variability : a review - Soil Ferti., 34 p., 1-15.

Bouchon J., Nys C., Ranger J., 1985. Cubage, biomasse et minéralomasse : comparaison de trois taillis simples des Ardennes primaires. - Acta Oecologica - Oecol. Plant, Vol. 6, (20), $\mathrm{n}^{\circ} 1,53-72$.

COMERFord N., LeAF A., 1982. An evaluation of techniques for sampling forest tree nutrient content. Part I : sampling the crown for total nutrient content. For. Sci., Vol. 28, $\mathrm{n}^{\circ} 3,471-480$.

Comerford N., LeAF A., 1982. An evaluation of techniques for sampling forest tree nutrient content. Part. Il : sampling for stem nutrient content. For. Sci., Vol. 28, n ${ }^{\circ}$, $481-487$.

CupI F., 1980. Les biomasses forestières, revue bibliographique 1950-1981. CNRF-INRA, 54280 Champenoux, France.

Drtessche R. Van Den, 1974. Prediction of mineral nutrient status of trees by foliar analysis. The botanical review. Vol. 40, n" 3, 347-394.

SNedecor G., Cochran W., 1967. Statistical methods. The Jowa State University press, Ames, Iowa, U.S.A.

Young H., CARPENTER P., 1976. Sampling variation of nutrient element content within and between trees of the same species. University of Maine at Orono, U.S.A. 